Check for updates

Cite this: RSC Adv., 2019, 9, 10360

Accepted 29th March 2019

DOI: 10.1039/c9ra01974j

rsc.li/rsc-advances

\section{Circularly polarized light modulated supramolecular self-assembly for an azobenzene- based chiral gel $\uparrow$}

\author{
Kenan Shao, ${ }^{\text {ab }}$ Ziyu Lv, ${ }^{c}$ Yuting Xiong, ${ }^{b}$ Guodong Li, ${ }^{\text {ab }}$ Dongdong Wang, \\ Haining Zhang $\left(\mathbb{D}^{\mathrm{a}}\right.$ and Guangyan Qing (D) *b
}

Modulating the self-assembling process and its nanoarchitecture by external stimuli has long been a challenging topic in supramolecular self-assembly. ${ }^{1}$ Research ranging from the fabrication of responsive supramolecules to the dynamic control of self-assembly behaviors has promoted broad and interesting applications in nanotechnology, ${ }^{2}$ electronics, ${ }^{3}$ tissue engineering ${ }^{4}$ and biomedical fields. ${ }^{5}$ Numerous exterior factors could affect self-assembly behaviors, such as temperature, solvent, magnetic field and light irradiation. ${ }^{6}$ Among these stimuli, light is considered to be remotely and accurately controlled, quickly switched and easily focused ${ }^{7}$ thus has attracted great interest in the construction of photo-responsive assemblies and devices.

Mainstreams of photo-responsive systems commonly focus on natural light. With further studies, investigations have been extended to polarized light, streams of photons with either right- or left-handed spin, which can transfer integer photonic spin to molecules. Such properties have endowed CPL as the inherent chiral light and has been regarded as the possible source of chiral information in living organisms. ${ }^{8}$ As exciting examples, CPL-driven absolute asymmetric (AAS) and mirrorsymmetry breaking (MSB) have been actively investigated in recent years; ${ }^{9}$ nanoparticles with chiral structures or helical arrangements could be generated by the CPL irradiation. ${ }^{10} \mathrm{By}$ comparison, the effect of CPL handedness on small molecule

${ }^{a}$ State Key Laboratory of Advanced Technology for Materials Synthesis and Processing, Wuhan University of Technology, 122 Luoshi Road, Wuhan 430070, P. R. China

${ }^{b}$ Key Laboratory of Separation Science for Analytical Chemistry, Dalian Institute of Chemical Physics, Chinese Academy of Sciences, 457 Zhongshan Road, Dalian 116023, P. R. China. E-mail: qinggy@dicp.ac.cn

${ }^{c}$ College of Electronic Science and Technology, Shenzhen University, Shenzhen 518060, P. R. China

$\dagger$ Electronic supplementary information (ESI) available. See DOI: $10.1039 / \mathrm{c} 9 \mathrm{ra} 01974 \mathrm{j}$ self-assembly is still with less exploration, especially those with photo-responsive capacities. In general, trans-azobenzene based gelators have strong self-assembly capacities owing to their favourable planar and symmetric structures. UV-light irradiation-induced trans-to-cis transition normally will break such molecular symmetry and lead to the collapse of selfassembled structures. Interestingly, in this study, we find that the introduction of CPL with specific handedness breaks this traditional idea (Fig. 1a). For an azobenzene-based chiral gel with $C_{2 \mathrm{v}}$-symmetric structure, right-CPL promotes the collapse of gel; by contrast, left-CPL triggers the formation of a new selfassembled structure, and macroscopic gel is well maintained. This unconventional finding affords a new pathway to the fabrications of photo-responsive devices.

Chemical structure of our chiral gelator, 4,4'-azobenzenelinked L-aspartate---phenylalanine methyl ester (abbreviated to Azo-DF), is shown in Fig. 1b. The 4,4'-di-substituted azobenzene, which supplies a $C_{2 \mathrm{v}}$-symmetric skeleton, functions as a photoresponsive group. While two L,L-DF dipeptides are linked to the two sides of azobenzene owing to their satisfactory self-assembly capacities. ${ }^{11}$ Synthesis process and characterization data of AzoDF are described in the ESI. $\uparrow$ An additional control experiment and the possible self-assembly modes calculated from quantum chemical calculation indicated that $\pi-\pi$ stacking between adjacent phenyl groups or between azo-benzene groups might be the driving force for the supramolecular self-assembly of Azo-DF (Fig. S3 and S4 in ESI †े). Owning to the excellent gelation capacity in chloroform/methanol $(\mathrm{v} / \mathrm{v}=1: 3),{ }^{12}$ our following research mainly focused on this mixed solvent (Table S1 in ESI†). The CPL pumping platform was consisted of an LED-UV (365 nm) light torch (SCOUT UVFLUXS-3W, $20 \mathrm{~mW} \mathrm{~cm} \mathrm{~cm}^{-2}$ ), a GlanThompson prism (200-900 nm) and a quarter-wave plate (Fig. 2a). Details on the CPL pumping platform are described in Part S4 in ESI. $\dagger$ Because of subtle changes under $3 \mathrm{~mW} \mathrm{~cm}^{-2}$ (distance $(D)$ : 

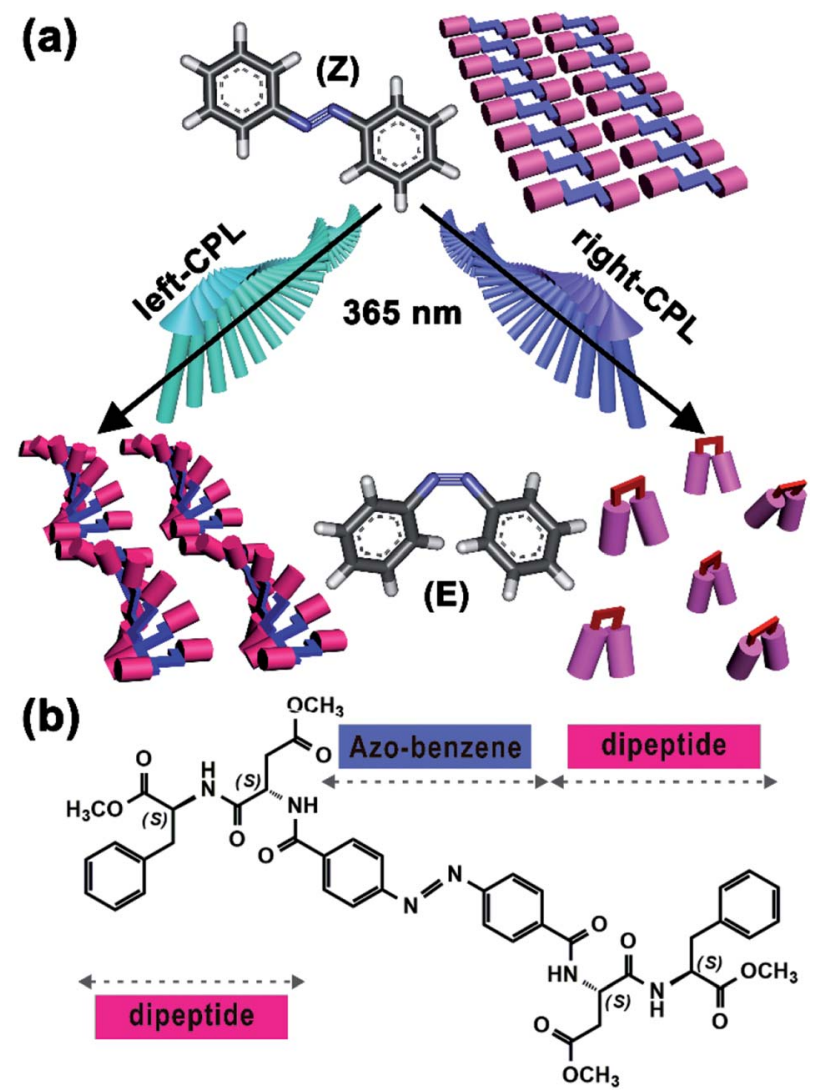

Fig. 1 (a) Illustration of different supramolecular self-assembly modulated by CPL handedness, right-CPL promotes the collapse of ordered structure, but left-CPL triggers the formation of new helical structure. (b) Chemical structure of $4,4^{\prime}$-azobenzene-linked dipeptide gelator, abbreviated to Azo-DF.

$45 \mathrm{~cm}$ ) light irradiation and too swift response to catch under 10 $\mathrm{mW} \mathrm{cm} \mathrm{cm}^{-2}(D: 10 \mathrm{~cm})$ irradiation, $5 \mathrm{~mW} \mathrm{~cm}^{-2}(D: 32.5 \mathrm{~cm})$ was regarded as the optimal light intensity in the following experiments. Meanwhile, the light intensities of left- and right-CPL were identical (Fig. S5b and c in ESI $\dagger$ ).

First, the photo-responsiveness of Azo-DF triggered by rightor left-CPL was detected by UV-vis absorption spectra. Azo-DF gelator was dissolved in chloroform at a low concentration of $0.05 \mathrm{mg} \mathrm{ml}^{-1}$, in which Azo-DF was in isolated state and supramolecular self-assembly would not happen. Initially, the UV-vis spectrum of Azo-DF consisted of a strong UV band with a maximum absorption peak at $326 \mathrm{~nm}$, which could be ascribed to the $\pi-\pi^{*}$ transition and corresponds to the vibrational structure of the typical trans-azobenzene (Fig. 2b). After irradiation of right- or left-CPL, two well separated bands in the UV region $\left(\lambda_{\max }\right.$ $\sim 290 \mathrm{~nm})$ and visible region $\left(\lambda_{\max } \sim 440 \mathrm{~nm}\right)$ increased, representing the $\pi-\pi^{*}$ and $n-\pi$ transition of cis-azobenzene structure, respectively. ${ }^{13}$ The photo-stationary state was reached after 30 min right- or left-CPL irradiation, revealing that the typical trans- to cis-transformation of azobenzene in Azo-DF had indeed happened. It is worth noting that the amount of range caused by CPL handedness were almost equal within the error range. A consistent tendency was observed when the individual azobenzene was irradiated by the left- or right-CPL (Fig. S6 in ESI $\dagger$ ).
Then the gelator concentration was increased to $2 \mathrm{mg} \mathrm{ml}^{-1}$ in chloroform/methanol $(\mathrm{v} / \mathrm{v}=1: 3)$, allowing the gel formation. The distinct gel-sol transition degree caused by the CPL handedness on macroscopic gel was observed. As shown in Fig. 2c, when the gel was exposed to the right-CPL irradiation, the gel showed an obvious tendency to loose and collapse after $3 \mathrm{~h}$ irradiation, remaining some fluffy aggregates in the $30 \mu \mathrm{L}$ solvents. By comparison, Azo-DF gel maintained stable in the gel state after $3 \mathrm{~h}$ of left-CPL irradiation, only a few solvents (12 $\mu \mathrm{L}$ ) were squeezed out. A similar solvent squeezed out situation of azobenzene gel, where a stable layered superstructure after UV light irradiation was claimed to be responsible, was reported by Jeong. ${ }^{14}$ Scanning electronic microscopy (SEM) were utilized to observe the gel morphology before and after $3 \mathrm{~h}$ of right- or left-CPL irradiation, respectively (Fig. 2d-f). Many long and regular ribbon-like fibres were observed. These fibres intertwined with each other and formed a dense three-dimensional (3D) network, revealing strong self-assembly capacities and linear packing pattern of Azo-DF. Large-scaled fibre distribution and enlarged view of a single fibre are shown in Fig. S7 in ESI. $\dagger$ After $3 \mathrm{~h}$ of right-CPL irradiation, the ribbon-like fibres were divided into numerous short stubs that scattered and piled up randomly, corresponding to the collapse of the gels. While after $3 \mathrm{~h}$ left-CPL irradiation, the intertwined ribbon-fibres were wellpreserved. The arrangement of fibres became slightly loose, which explained why there some solvents were squeezed out.

Circular dichroism (CD) spectroscopy was used to monitor this self-assembly process (Fig. $2 \mathrm{~g}$ and $\mathrm{h}$ ). Initially, there are two weak peaks at 260 and $330 \mathrm{~nm}$ in the CD spectrum, which could be attributed to the molar chirality of two dipeptide arms carried by Azo-DF gelator itself. Such weak peaks were consistent with the morphology observed by SEM, where no evidential helical structure was detected. When the Azo-DF gel was irradiated with right-CPL, fluctuations around the baseline were observed in the CD spectra. But with the left-CPL irradiation, the CD spectrum (Fig. 2h) shows negative and positive cotton effect bands at 314 and $385 \mathrm{~nm}$, respectively. These bands enhanced remarkably after 3 hours of the left-CPL irradiation, indicating the appearance of new helical structure during selfassembly process under the left-CPL irradiation, ${ }^{15}$ although such helical structure was not a common scenario.

$\mathrm{X}$-ray powder diffraction (XRD) was implemented to further reveal the stacking structures of the xerogel. Fig. 3a shows XRD spectrum of Azo-DF xerogel before CPL irradiation, multiple sharp peaks indicated long-range molecular packing and high crystallinity of the gel structure. ${ }^{16}$ After r-CPL irradiation for $3 \mathrm{~h}$, the measured XRD signals sharply diminished to bottom line (Fig. 3b), which indicated that the trans-to-cis transition of the azobenzene obviously decreased the planar symmetry of the gelators, destroying the highly ordered stacking of the gelators. This phenomenon were well consistent with the morphological changes observed by SEM. By comparison, for the gel sample after left-CPL illumination for $3 \mathrm{~h}$, the crystal order was well maintained except some slight decreases in the XRD intensity. In addition, several new diffraction peaks are observed in Fig. 3c. Specifically, the peak A $\left(2 \theta=7.81^{\circ}\right)$ splits into two bands, while two new peaks $\left(\mathrm{B}^{\prime}\right.$ and $\left.\mathrm{C}^{\prime}\right)$ are found at $2 \theta=1.66^{\circ}$, 

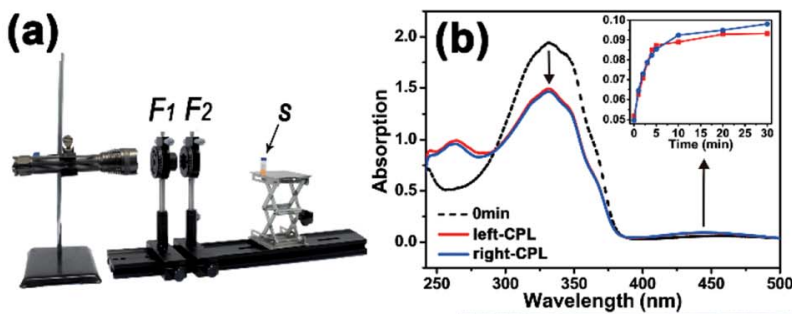

(c)
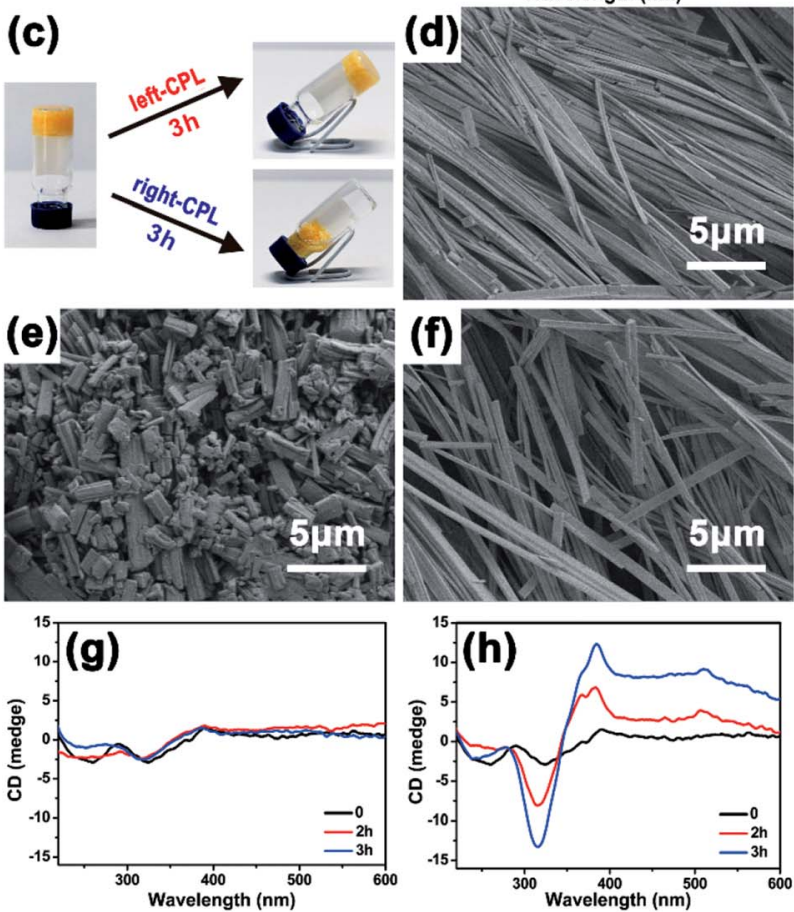

Fig. 2 (a) Photo of the CPL pumping system. F1: Glan-Thompson prism; F2: quarter-wave plate; s: gelator sample. (b) UV-vis spectra of Azo-DF in chloroform $\left(0.05 \mathrm{mg} \mathrm{ml}^{-1}\right.$ ) before (dotted line) and after (solid lines) CPL illumination for $30 \mathrm{~min}$. (c) Gel-sol transition of Azo-DF in chloroform/methanol ( $\left.\mathrm{v} / \mathrm{v}=1: 3,2 \mathrm{mg} \mathrm{ml}^{-1}\right)$, the gel collapsed under right-CPL irradiation, compared to only few solvent squeezing out from the gel under left-CPL irradiation. ( $d-f)$ Scanning electronic microscopy (SEM) images of Azo-DF xerogel before (d) and after $3 \mathrm{~h}$ irradiation of right-CPL (e) or left-CPL (f). ( $g$ and h) Circular dichroism (CD) spectra of Azo-DF gel in chloroform/methanol ( $\left.\mathrm{v} / \mathrm{v}=1: 3,2 \mathrm{mg} \mathrm{m}^{-1}\right)$ before and after the irradiation of right-(g) or left-CPL (h). ( $\lambda: 365 \mathrm{~nm}, 5 \mathrm{~mW} \mathrm{~cm}{ }^{-2}$ ).

$7.90^{\circ}$. In wide-angle region $\left(2 \theta=18-25^{\circ}\right)$ the position of diffraction peak appears a shift, ${ }^{16}$ while the peak D splits into two peaks and the peak $\mathrm{E}$ decreases remarkably. These data clearly suggested that new crystalline state came into being after left-CPL illumination, moreover, the decomposition of the selfassembled structure might be largely postponed by left-CPL.

Unambiguous evidence for CPL handedness-dependent supramolecular self-assembly was provided by atomic force spectroscopy (AFM). Optimization experiments indicated that the Azo-DF could form highly ordered self-assembled pattern at a concentration of $0.5 \mathrm{mg} \mathrm{ml}^{-1}$ in chloroform. So we prepared AFM sample by dripping one drop of Azo-DF solution $(0.5 \mathrm{mg}$ $\mathrm{ml}^{-1}$, chloroform) before and after right- or left-CPL irradiation onto freshly cleaved mica, respectively. As shown in Fig. 4a, the initially self-assembled pattern of Azo-DF presents as numerous short nanofibers evenly distributed in three directions, the

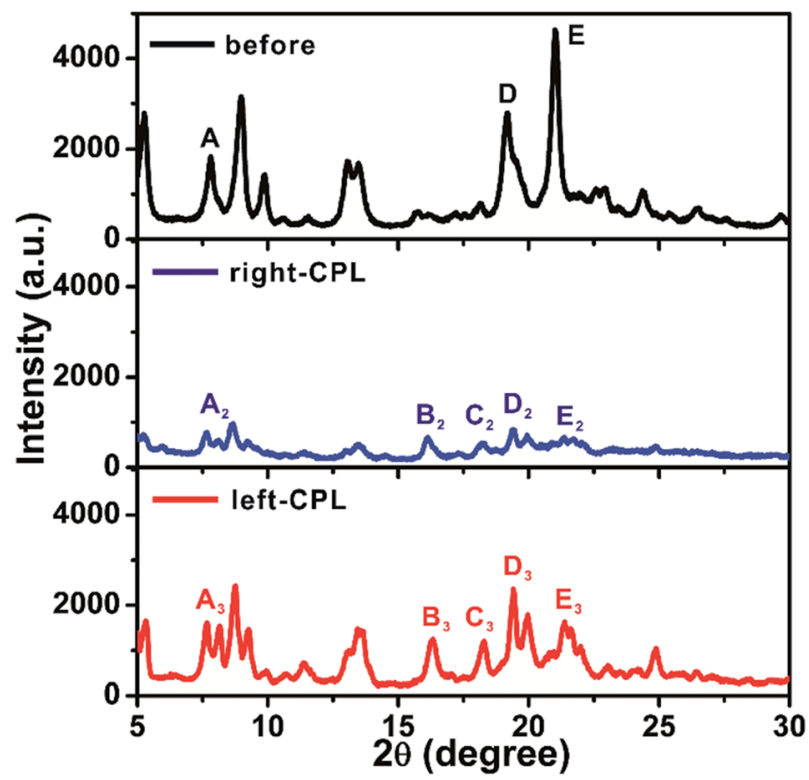

Fig. 3 XRD spectra of Azo-DF xerogels before and after $3 \mathrm{~h}$ irradiation of right- or left-CPL, light intensity: $5 \mathrm{~mW} \mathrm{~cm}^{-2}$.

average length, width and height of these nanofibers are $2.4 \mu \mathrm{m}$, $80 \mathrm{~nm}$ and $25 \mathrm{~nm}$, respectively. Upon the irradiation of rightCPL for $2 \mathrm{~h}$, the three-direction short nanofibers are replaced by some long and flattened ribbons with average length of more than $10 \mu \mathrm{m}$ and height of $30 \mathrm{~nm}$ (Fig. $4 \mathrm{~b}$ ). After $3 \mathrm{~h}$ right-CPL irradiation, the self-assembled structure was hard to be detected (Fig. 4c). Such obvious change could be reasonably attributed to the weak self-assembled capacity of the gelator in a cisazobenzene form, which dominated the configuration of the gelator. In comparison, when Azo-DF was irradiated by left-CPL for $2 \mathrm{~h}$ (Fig. 4d), rather than decomposition, those short nanofibers showed a strong tendency to aggregate, the fiber length and width were estimated to be $3.8 \mu \mathrm{m}$ and $40 \mathrm{~nm}$. A detailed enlarged view (Fig. 4e) of $1 \mu \mathrm{m}$ shows that these nanofibers intertwisted with each other to form a rope like structure. More interestingly, when the Azo-DF solution was exposed to left-CPL for $3 \mathrm{~h}$ (Fig. 4f), a dendritic structure was observed featured with an aggregate center over $150 \mathrm{~nm}$ height and numerous branches of nearly $50 \mathrm{~nm}$ height. The aggregation difference resulted from the CPL handedness was also monitored by dynamic light scattering (DLS) measurement (Fig. S8 in ESI†). The distinct self-assembly difference was well consistent with the above gel morphological changes. At the same time, the subsequent fiber growth upon left-CPL irradiation further demonstrated a special staking model of Azo-DF gelators where the supramolecular self-assembly was successfully inverted by exchanging the handedness of CPL. A nearly reversed CPL-modulated self-assembly trend was detected by using Azo-D,D-DF (Fig. S9 in ESI†). Considering Azo-L,L-DF and Azo-D,D-DF were not completely mirror symmetric owing to the four chiral centers, this result provided an auxiliary proof that the distinct self-assembly behaviors of the gelators were caused by CPL handedness. Compared to natural light, the introduction of CPL significantly emphasized the quantum characteristic of light orientation, ${ }^{8 a}$ where the delicate balance between 


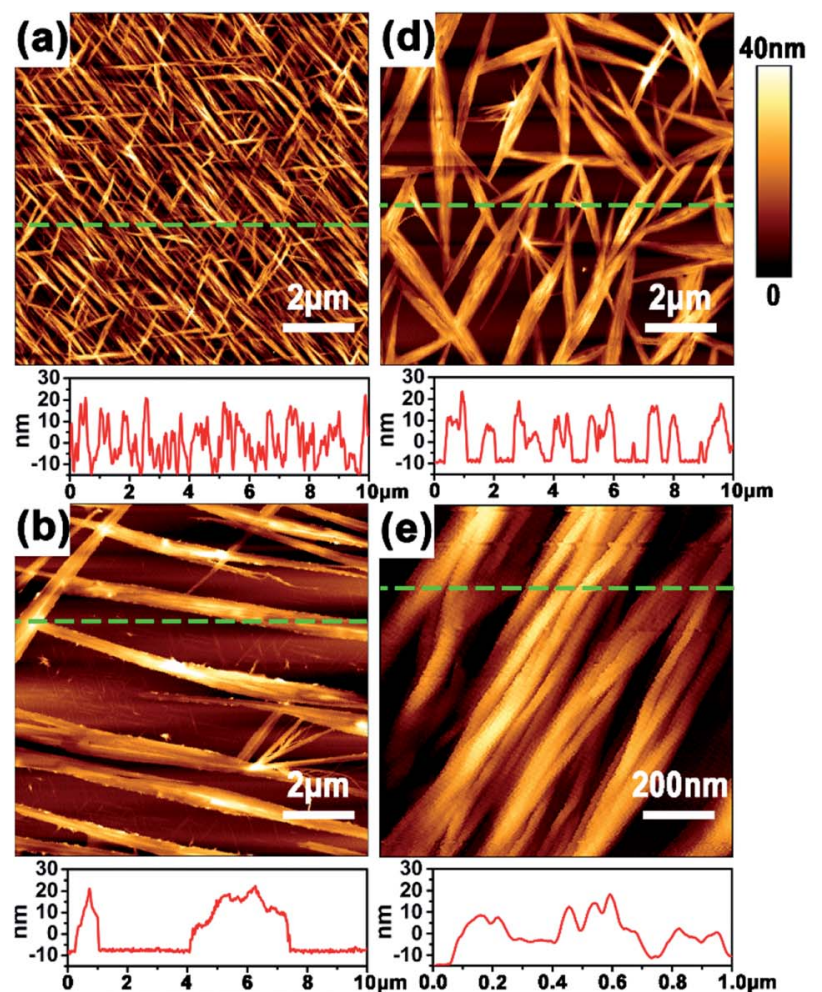

(c)

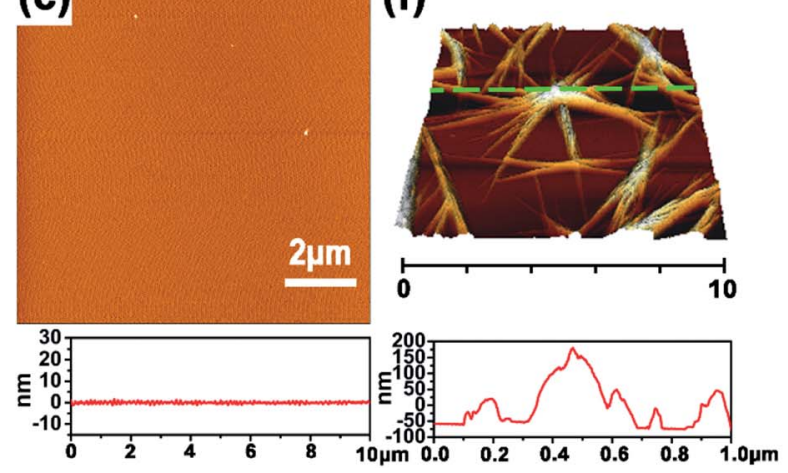

Fig. 4 AFM images of the self-assembled morphologies and the corresponding section profiles of Azo-DF in chloroform $\left(0.5 \mathrm{mg} \mathrm{ml}^{-1}\right)$ before (a) and after $2 \mathrm{~h} \mathrm{(b)}$ or $3 \mathrm{~h}$ (c) of right-CPL irradiation; (d-f) AzoDF after $2 \mathrm{~h}$ ( $\mathrm{d}$ and e) or $3 \mathrm{~h}(\mathrm{f})$ of left-CPL irradiation.

the isomerization of azobenzene and the chiral preference of the dipeptide played an important role in the dissociation or supramolecular self-assembly of Azo-DF gelators. ${ }^{13 b, 17}$ The possible mechanism is described in Part S6 in ESI. $\dagger$

In conclusion, we observed an unconventional chiral effect: azobenzene-based chiral gelator showed a collapse tendency under the right-CPL irradiation, by contrast, intensive selfassembly emerged when the gelator was irradiated with leftCPL. Such CPL handedness-triggered self-assembly difference might arise great interest in the potential of CPL sources in controlling the supramolecular self-assembly of chiral molecules or polymers as well as for exploring their roles in the fabrication of function materials, moreover, inspiring rethought of some vital chemical and biological processes from the unique perspective of "dynamic molecular chirality".

\section{Conflicts of interest}

There are no conflicts to declare.

\section{Acknowledgements}

This work was supported by the National Natural Science Foundation of China (51473131, 51533007 and 21775116), DICP Innovation Funding (DICP-RC201801). G. Qing acknowledges Wuhan Morning Light Plan of Youth Science and Technology.

\section{Notes and references}

1 (a) L. E. Buerklea and S. J. Rowan, Chem. Soc. Rev., 2012, 41, 6089; (b) E. R. Draper, E. G. B. Eden, T. O. McDonald and D. J. Adams, Nat. Chem., 2015, 7, 848.

2 D. M. Raymond and B. L. Nilsson, Chem. Soc. Rev., 2018, 47, 3659 .

3 X. Feng, V. Marcon, W. Pisula, M. R. Hansen, J. Kirkpatrick, F. Grozema, D. Andrienko, K. Kremer and K. Müllen, Nat. Mater., 2009, 8, 421.

4 C. J. Bruns and J. F. Stoddart, Acc. Chem. Res., 2014, 47, 2186.

5 I. V. Kolesnichenko and E. V. Anslyn, Chem. Soc. Rev., 2017, 46, 2385.

6 (a) S. Dai, P. Ravi and K. C. Tam, Soft Matter, 2009, 5, 2513; (b) N. Ousaka, Y. Takeyama and E. Yashima, Chem. Sci., 2012, 3, 466.

7 F. Ercole, T. P. Davis and R. A. Evans, Polym. Chem., 2010, 1, 37.

8 (a) M. Padgett and R. Bowman, Nat. Photonics, 2011, 5, 343; (b) C. Giri, F. Goesmann, C. Meinert, A. C. Evans and U. J. Meierhenrich, Top. Curr. Chem., 2013, 333, 41.

9 L. Wang, L. Yin, W. Zhang, X. Zhu and M. Fujiki, J. Am. Chem. Soc., 2017, 139, 13218.

10 Y. Tang and A. E. Cohen, Science, 2011, 332, 333.

11 (a) Y. Lin, Y. Qiao, P. Tang, Z. Lib and J. Huang, Soft Matter, 2011, 7, 2762; (b) T. M. Doran, D. M. Ryan and B. L. Nilsson, Polym. Chem., 2014, 5, 241.

12 Z. Chen, Z. Lv, G. Qing and T. Sun, J. Mater. Chem. B, 2017, 5, 3163.

13 (a) C. L. Forber, E. C. Kelusky, N. J. Bunce and M. C. Zerner, J. Am. Chem. Soc., 1985, 107, 5884; (b) T. Schultz, J. Quenneville, B. Levine, A. Toniolo, T. J. Martínez, S. Lochbrunner, M. Schmitt, J. P. Shaffer, M. Z. Zgierski and A. Stolow, J. Am. Chem. Soc., 2003, 125, 8098.

14 Y. J. Choi, J. T. Kim, W. J. Yoon, D. G. Kang, M. Park, D. Y. Kim, M. H. Lee, S. k. Ahn and K. U. Jeong, ACS Macro Lett., 2018, 7, 576.

15 G. Gottarelli, S. Lena, S. Masiero, S. Pieraccini and G. P. Spada, Chirality, 2008, 20, 471.

16 J. Cui, Y. Zheng, Z. Shen and X. Wan, Langmuir, 2010, 26, 15508.

17 (a) L. Nikolova, L. Nedelchev, T. Todorov, T. Petrova, N. Tomova and V. Dragostinova, Appl. Phys. Lett., 2000, 77, 657; (b) D. K. Hore and A. L. Natansohn, J. Phys. Chem. B, 2003, 107, 2197; (c) S. W. Choi, S. Kawauchi, N. Y. Ha and H. Takezo, Phys. Chem. Chem. Phys., 2007, 9, 3671. 